\title{
Scabies, incomplete lupus erythematosus and Henoch-Schonlein purpura
}

Yang Fang $\mathrm{Wu}^{1}$, Jing Jing Wang ${ }^{2}$, Hui Hui Liu ${ }^{1}$, Wei Xia Chen ${ }^{1}$, Peng Hu${ }^{1}$

\author{
'Department of Pediatrics, the First Affiliated Hospital of Anhui Medical University, \\ Hefei, China \\ Jjinling Hospital, Nanjing University School of Medicine, Nanjing, China
}

Submitted: 4 October 2020; Accepted: 9 December 2020;

Online publication: 26 February 2021

Arch Med Sci 2021; 17 (2): 564-568

DOI: https://doi.org/10.5114/aoms/131542

Copyright @ 2021 Termedia \& Banach

Henoch-Schonlein purpura (HSP) is an immunoglobulin A (IgA)-mediated, autoimmune, hypersensitivity vasculitis in childhood, mainly characterized by non-thrombocytopenic purpura, abdominal pain, arthritis and renal involvement. An epidemiological survey in Taiwan, China from 1997 to 2012 that recruited a total of 1002 HSP children demonstrated an annual incidence of 9.61 per 100,000 children younger than 18 years. The occurrence of HSP peaks between 7 and 9 years of age, and male children are more often affected than female children [1, 2]. Currently, the detailed etiology of HSP remains unknown. Several triggers, including infectious agents, foods, drugs, vaccinations and insect bites, have been reported to be associated with HSP onset. Recently, our research panel retrospectively reviewed the clinical records of 1200 HSP children, and found that Streptococcus was the most frequent infectious trigger and was identified in 205 cases (17.08\%), followed by Helicobacter pylori, Mycoplasma (MP), parainfluenza, respiratory syncytial virus, Mycobacterium tuberculosis and Toxoplasma gondii [2]. Scabies is a skin hypersensitivity reaction caused by infestation with the mite Sarcoptes scabiei var. hominis, and its prevalence is decreasing worldwide with the improvement of environmental hygiene [3]. In the present report, we encountered an unusual patient who suffered from HSP and incomplete lupus erythematosus (ILE) on day 8 after scabies. ILE patients have at least one but less than four of the American College of Rheumatology (ACR) classification criteria of systemic lupus erythematosus (SLE) and do not present distinctive clinical features or meet classification criteria of other connective tissue diseases [4].

An 11-year-old boy was first referred to the Department of Dermatology in November 2018, with a history of nocturnal and generalized pruritus, and itchy rashes on his upper trunk. Physical examination revealed multiple small $(<6 \mathrm{~mm})$ papules and raised burrows on his upper trunk. By a light microscope under low power, a translucent mite around 0.2 to $0.5 \mathrm{~mm}$ long with four pairs of brown legs was seen on the direct examination of skin scrapings (Figure $1 \mathrm{~A}$ ). He was diagnosed with scabies and treated with whole-body applications of $5 \%$ permethrin lotion and oral cetirizine $(10 \mathrm{mg} /$ day). On day 8 , the papules and small burrows were significantly resolved, whereas edema and palpable purpuric skin lesions were found on his lower limbs (Figure 1 B), combined with transient abdominal pain. Under the circumstances, he was brought to our department for a further consultation. On admission, his blood pressure

\author{
Corresponding author: \\ Peng Hu PhD \\ Department of Pediatrics \\ the First Affiliated \\ Hospital of \\ Anhui Medical University \\ Hefei, China \\ Phone: +86055162922058 \\ E-mail: hupeng28@aliyun.com
}



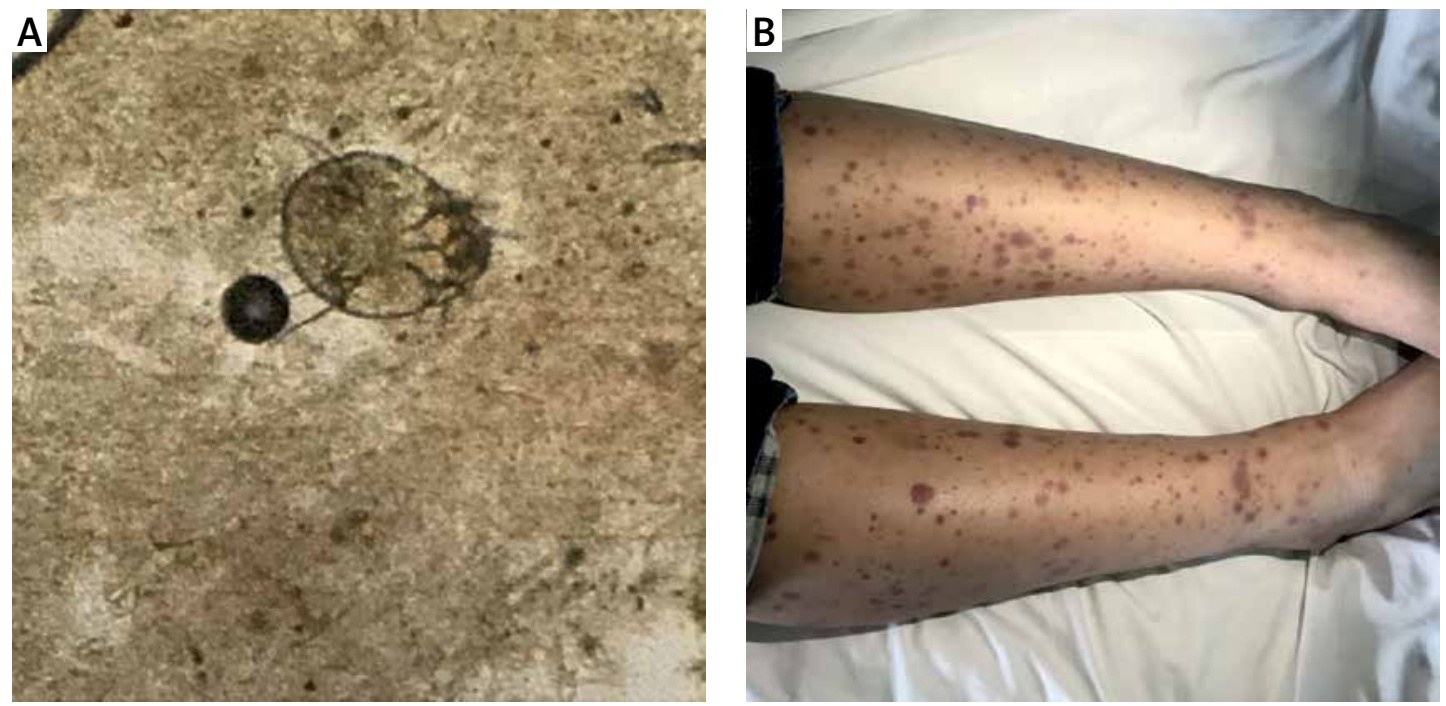

C
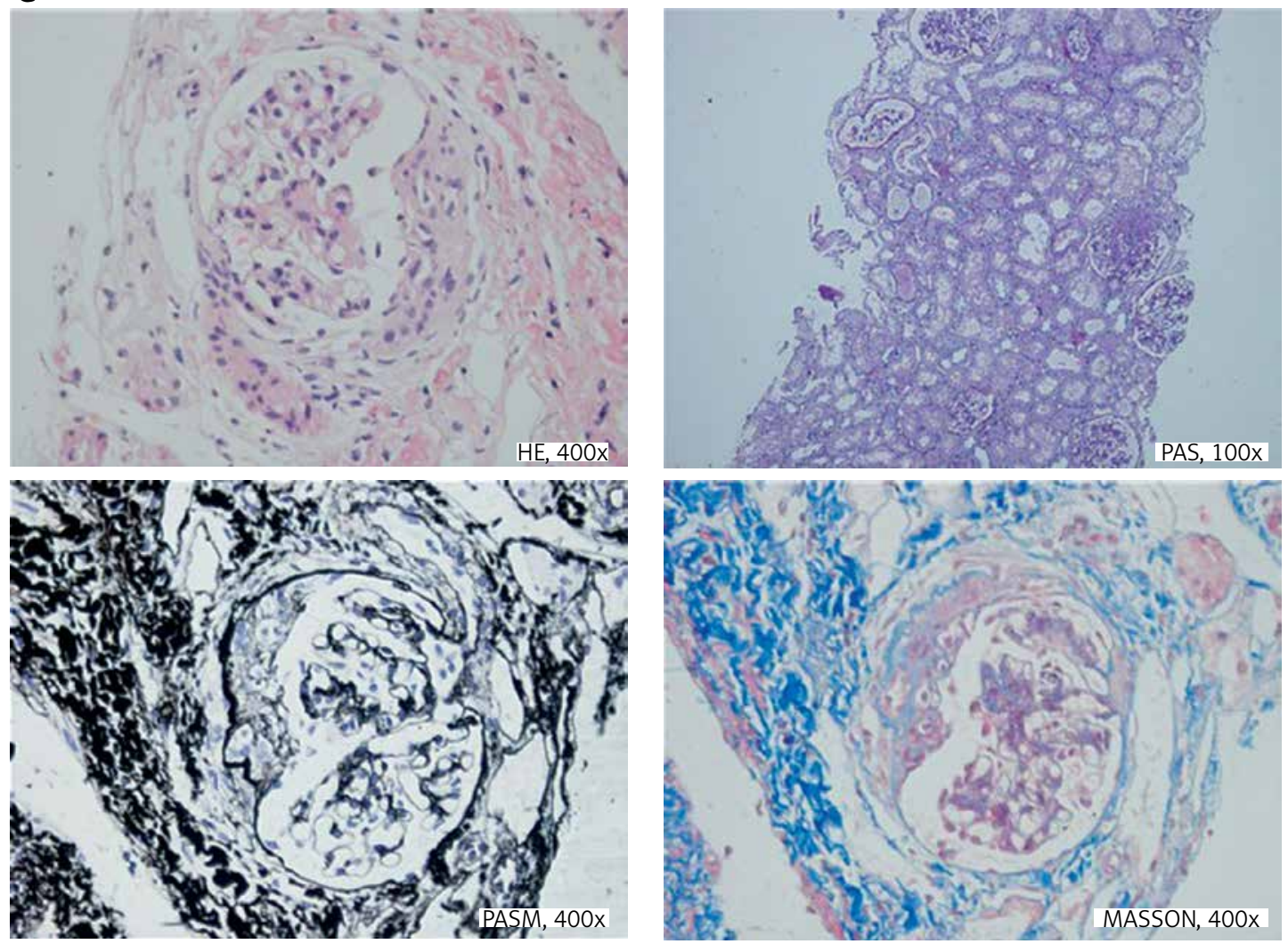

Figure 1. Skin scrapings, manifestations, renal pathology and immunofluorescence. A - Skin scrapings revealed translucent mite around 0.2 to $0.5 \mathrm{~mm}$ long with four pairs of brown legs. B - Palpable purpura symmetrically distributed in the lower limbs. C - Histopathological findings of the renal biopsy specimens showed 8 glomeruli, one with evidence of global sclerosis, and crescent formation was observed in 5/8 glomeruli. Mild mesangial proliferation and expansion were also seen in glomeruli. Moreover, mild expansion was noted in the interstitium, in association with tubular atrophy. The patient was diagnosed as having HSP nephritis (International Study of Kidney Disease in Children category: Class IIIb)

was 108/80 $\mathrm{mm} \mathrm{Hg}$, heart rate was 65 beats/min, axillary temperature was $36.8^{\circ} \mathrm{C}$ and respiratory rate was 18 breaths/min. Except for the characteristic purpura and periumbilical tenderness, the cardio-pulmonary examination was unremarkable. His initial blood counts were as follows: white blood cells $6.68 \times 10^{9} /$, basophils $0.10 \%$, red blood cells $5.01 \times 10^{12} /$, hemoglobin $154 \mathrm{~g} / \mathrm{l}$, and platelets $221 \times 10^{9} /$. Other blood chemistry findings were erythrocyte sedimentation rate $10 \mathrm{~mm} / \mathrm{h}$, C-reactive protein $0.80 \mathrm{mg} / \mathrm{l}$, creatinine $63 \mu \mathrm{mol} / \mathrm{l}$, serum ferritin $20.61 \mu \mathrm{g} / \mathrm{l}$, complement 3 (C3) $1.12 \mathrm{~g} / \mathrm{l}$, complement 4 (C4) $0.24 \mathrm{~g} / \mathrm{l}$ and IgA $2.88 \mathrm{~g} / \mathrm{l}$. The autoimmune serologic workup came 
D
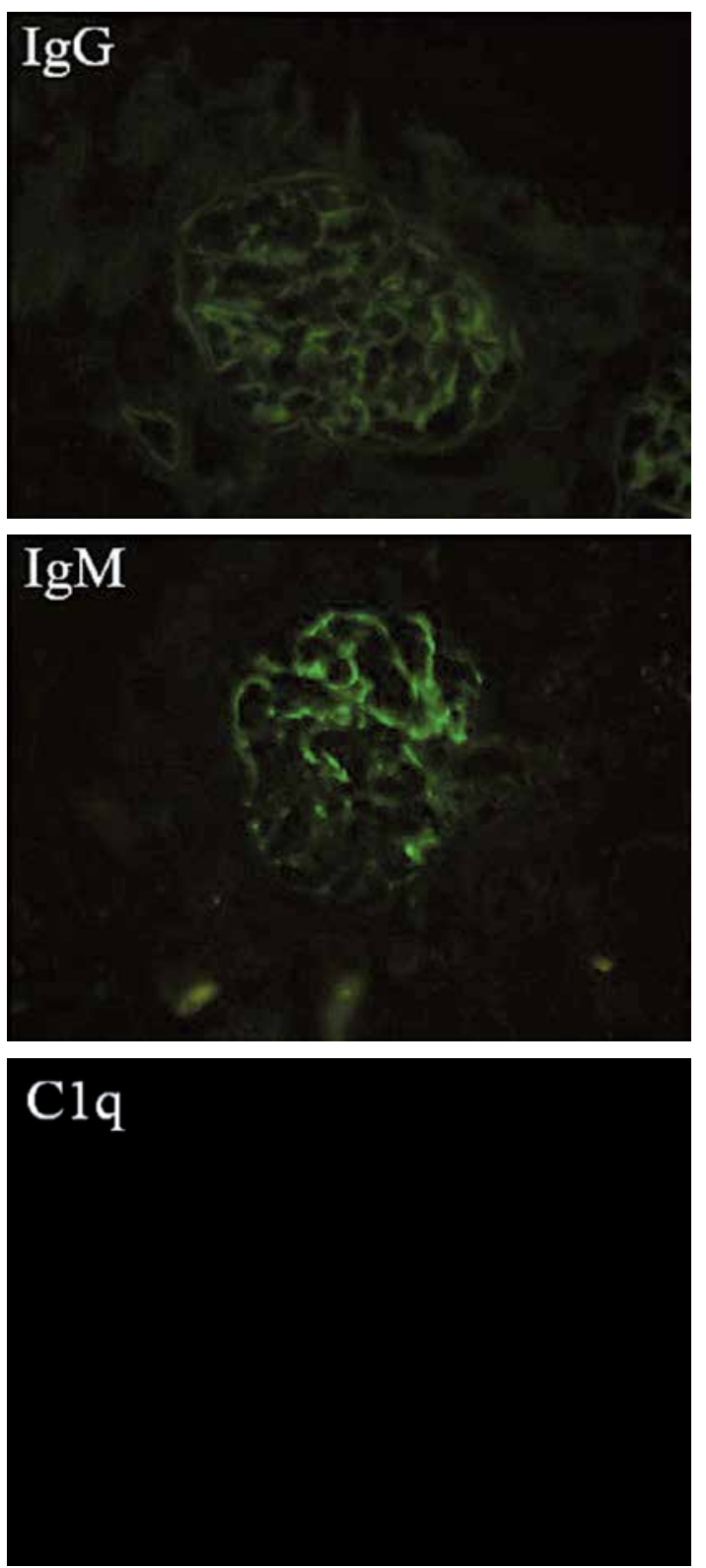
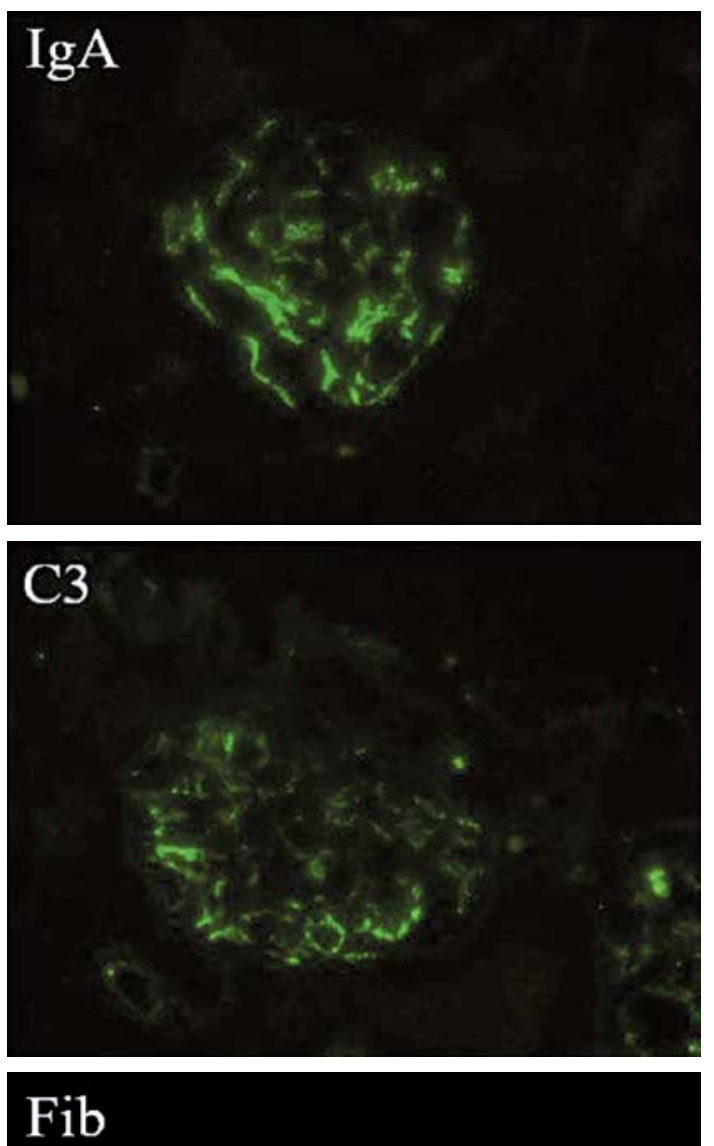

Figure 1. Cont. D - The immunofluorescence microscopy revealed granular deposits of IgA, IgM and C3 in the glomerular mesangium

back positive for antinuclear antibody (ANA); negative for anti-double stranded DNA antibody (anti-dsDNA), anti-Smith antibody (anti-SM) and anti-neutrophil cytoplasmic antibody (ANCA). Antibody tests for human immunodeficiency virus (HIV), hepatitis B virus (HBV), hepatitis C virus (HCV), Epstein-Barr virus (EBV), MP and Mycobacterium tuberculosis were also not reactive. Urinalysis showed red blood cells: $1+$, protein: $2+$ with $24 \mathrm{~h}$ urine protein of $0.90 \mathrm{~g}$. A routine stool test showed weak positive fecal occult blood but no erythrocytes. The electrocardiogram, abdominal and renal ultrasonography had no significant $a b$ normalities. According to the above clinical and laboratory findings, he was diagnosed as having
HSP and ILE. Taking into consideration medium proteinuria, a course of pulse methylprednisolone $(10 \mathrm{mg} / \mathrm{kg} /$ day) was administered for 6 days, followed by oral prednisone (1 $\mathrm{mg} / \mathrm{kg} /$ day). On day 18 , the purpuric rashes almost disappeared, whereas medium proteinuria still existed. A renal biopsy was performed and the histopathological examination of the biopsy specimen revealed 8 glomeruli with one showing evidence of global sclerosis, and crescent formation was observed in 5/8 glomeruli. Mild mesangial proliferation and expansion were also seen in glomeruli. Moreover, mild expansion was noted in the interstitium, in association with tubular atrophy (Figure $1 \mathrm{C}$ ). The immunofluorescence microscopy revealed granu- 
lar deposits of IgA, IgM and C3 in the glomerular mesangium (Figure $1 \mathrm{D}$ ). Based on these findings, he was diagnosed as having HSP nephritis (International Study of Kidney Disease in Children (ISKDC) category: Class IIIb). The patient was treated with high-dosage intravenous methylprednisolone $(30 \mathrm{mg} / \mathrm{kg} /$ day) followed by prednisone (1 $\mathrm{mg} / \mathrm{kg} /$ day) combined with oral mycophenolate mofetil $(20 \mathrm{mg} / \mathrm{kg} /$ day). The pulse therapy was performed three times in total and his proteinuria gradually decreased. The study protocol was approved by the Research Ethics Commission of the First Affiliated Hospital of Anhui Medical University.

In this report, we have described an unusual case of a patient who suffered from HSP on day 8 after scabies. As there is no definitive test for HSP diagnosis, the 2008 European League Against Rheumatism criteria are still the most commonly used guidelines for HSP diagnosis [5]. On this basis, our patient fulfilled 3 criteria (nonthrombocytopenic purpura, abdominal pain and renal involvement), and thus the diagnosis of HSP was made undoubtedly after scabies. To the best of our knowledge, very few previous studies have documented that cutaneous vasculitis can be secondary to or coexisting with scabies [6]. Furthermore, Liu et al. [7] tracked 4481 scabies patients for a 7-year period and found that scabies led to a 5.44-fold increased risk for hypersensitivity vasculitis, a 4.91-fold increased risk for dermatomyositis, a 2.89-fold increased risk for polyarteritis nodosa, and a 2.73-fold increased risk for systemic lupus erythematosus, compared with the healthy population. According to the current limited evidence, we speculated that the potential mechanisms of scabies-associated HSP may be attributed to the host response to antigens on Sarcoptes scabiei var. Hominis, such as immune-complex mediated injury, cytotoxic T-cell-mediated immune responses and autoimmune reactions. In addition, Ito et al. [8] detected basophil recruitment in skin lesions of both scabies and HSP, and pointed out that basophils served as a possible common element in their pathophysiological mechanisms of immunoregulation and microvascular activation. Further studies are warranted to clarify their shared etiologies and relationships.

As for the present case, another unique feature was the positive ANA, whereas the other diagnostic criteria for SLE were absent. In fact, HSP and SLE share several symptomatic overlaps, such as cutaneous lesions and multi-systematic involvement. In our previous report, we described an unusual female patient who suffered from HSP at 3 months after diagnosis of SLE. Unlike the present case, her autoimmune serologic workup indicated positive anti-dsDNA/anti-SM and low C3/C4 besides positive ANA [9]. ILE patients had at least one but less than four of the ACR classification criteria of SLE and did not present distinctive clinical features or meet classification criteria of other connective tissue diseases [4]. Although the majority of ILE patients may never reach SLE classification, about $10-50 \%$ of ILE patients are at high risk of progressing to complete SLE within 5 years [10]. In southern Sweden, Ståhl Hallengren et al. [11] followed up 28 ILE patients for a 10-year period and found that 16 of 28 patients developed complete SLE, and malar rash and anticardiolipin antibodies were potent predictors of complete SLE; in addition, positive Wasserman and ANA, thrombocytopenia, arthritis and early involvement of multiple organs could also serve as candidates of risk factors for development of complete SLE. Based on the experiences from adult ILE, antimalarial drugs may play a beneficial role in limiting organ damage, delaying complete SLE onset, slowing the accrual of autoantibodies and reducing the need for drugs with greater toxicity, but which have not yet been formally tested in longitudinal studies and even in pediatric patients [12].

During the period of hospitalization, our patient suffered from acute kidney injury and had persistent proteinuria. The histopathological examination of the renal biopsy specimen showed that the pathological classification was class IIIb. As we all know, the degree of proteinuria has been proven to be not only a sign but an accelerator of nephropathies. A recent single-center study from Shanghai Children's Hospital showed that the pathological classification in $62.97 \%$ of HSP patients with nephrotic proteinuria was above ISKDC class III [13]. Currently, the main treatments of HSP nephritis encompass corticosteroids alone or plus immunosuppressors. Feng et al. [13] administered methylprednisolone and mycophenolate mofetil to 54 HSP patients with nephrotic proteinuria, and found that $68.52 \%$ had complete remission and no patient progressed to end-stage renal disease after a follow-up of 6 months to 5 years. In the present report, it should be noted that corticosteroids and immunosuppressors may increase the relapse risk of scabies. Therefore this patient should be followed up carefully after being discharged from our department.

In conclusion, in the present report, we describe, for the first time, an unusual patient who suffered from HSP and ILE on day 8 after scabies. Several triggers, especially for infectious agents, have been proved to be associated with HSP onset. In this circumstance, scabies may be considered as a potential trigger in our patient. In addition, the aim of ILE diagnosis in this case is to improve the clinicians' awareness of those patients who have a significant risk for progression to complete SLE during a long-term follow-up. 


\section{Acknowledgments}

Yang Fang Wu and Jing Jing Wang contributed equally to this work and should be considered cofirst authors.

\section{Conflict of interest}

The authors declare no conflict of interest.

\section{References}

1. Lei WT, Tsai PL, Chu SH, et al. Incidence and risk factors for recurrent Henoch-Schonlein purpura in children from a 16-year nationwide database. Pediatr Rheumatol Online J 2018; 16: 25.

2. Xu Y, Wang JJ, Liu FF, et al. Predisposing factors of childhood Henoch-Schonlein purpura in Anhui province, China. J Investig Med 2019; 67: 771-8.

3. Engelman D, Steer AC. Control strategies for scabies. Trop Med Infect Dis 2018; 3: 98.

4. Vilá LM, Mayor AM, Valentín AH, García-Soberal M, Vilá S. Clinical outcome and predictors of disease evolution in patients with incomplete lupus erythematosus. Lupus 2000; 9: 110-5.

5. Ozen S, Pistorio A, Iusan SM, et al. EULAR/PRINTO/PRES criteria for Henoch-Schonlein purpura, childhood polyarteritis nodosa, childhood Wegener granulomatosis and childhood Takayasu arteritis: Ankara 2008. Part II: Final classification criteria. Ann Rheum Dis 2010; 69: 798-806.

6. Clevy C, Brajon D, Combes E, et al. Scabietic vasculitis: report of 2 cases. Ann Dermatol Venereol 2017; 144: 349-55.

7. Liu JM, Chiu FH, Lin CY, Chang FW, Hsu RJ. Incidence of autoimmune diseases in patients with scabies: a nationwide population-based study in Taiwan. Rheumatol Int 2017; 37: 1125-34.

8. Ito Y, Satoh T, Takayama K, Miyagishi C, Walls AF, Yokozeki $\mathrm{H}$. Basophil recruitment and activation in inflammatory skin diseases. Allergy 2011; 66: 1107-13.

9. Hu P, Huang BY, Zhang DD, et al. Henoch-Schönlein purpura in a pediatric patient with lupus. Arch Med Sci 2017; 13: 689-90.

10. Laustrup H, Voss A, Green A, Junker P. Occurrence of systemic lupus erythematosus in a Danish community: an 8-year prospective study. Scand J Rheumatol 2009; 38: 128-32.

11. Ståhl Hallengren C, Nived O, Sturfelt G. Outcome of incomplete systemic lupus erythematosus after 10 years. Lupus 2004; 13: 85-8.

12. Aberle T, Bourn RL, Munroe ME, et al. Clinical and serologic features in patients with incomplete lupus classification versus systemic lupus erythematosus patients and controls. Arthritis Care Res (Hoboken) 2017; 69: 1780-8.

13. Feng D, Huang WY, Hao S, et al. A single-center analysis of Henoch-Schonlein purpura nephritis with nephrotic proteinuria in children. Pediatr Rheumatol Online J 2017; 15: 15. 